\title{
Rate-dependent shear-band initiation in a metallic glass
}

\author{
D. Tönnies, ${ }^{1}$ K. Samwer, ${ }^{2}$ P. M. Derlet ${ }^{3}$ C. A. Volkert, ${ }^{1, a)}$ and R. Maaß ${ }^{1, b)}$ \\ ${ }^{1}$ Institute of Materials Physics, University of Göttingen, Friedrich-Hund-Platz 1, 37077 Göttingen, Germany \\ ${ }^{2}$ Ist Physics Institute, University of Göttingen, Friedrich-Hund-Platz 1, 37077 Göttingen, Germany \\ ${ }^{3}$ Condensed Matter Theory Group, Paul Scherrer Institute, 5232 Villigen, Switzerland
}

(Received 1 March 2015; accepted 16 April 2015; published online 28 April 2015)

\begin{abstract}
A statistical analysis of the rate-dependent pop-in behavior during spherical nanoindentation of a metallic glass is presented. Since the first pop-in of a test corresponds to the initiation of plasticity, this method provides insights into the fundamentals of shear-banding behavior. In this study, test series with different applied loading rates reveal that the initiation of a shear band is thermally activated with a rate- and stress-dependent activation volume. We further find a spatial inhomogeneity of the elastic modulus at the micrometer scale. This long-range structural heterogeneity does not strongly correlate with the shear-band initiation stress. (C) 2015 AIP Publishing LLC.

[http://dx.doi.org/10.1063/1.4919134]
\end{abstract}

Amorphous metals, also known as metallic glasses (MGs), are known to exhibit remarkably high elastic limits prior to the onset of plastic deformation. ${ }^{1}$ Upon yielding strongly localized planar defects are formed. These defects are called shear bands and have a core-thickness of some tens of nanometers, ${ }^{2}$ may have a micro-meter softened zone, ${ }^{3}$ and can span the entire bulk piece of $\mathrm{mm}$ dimensions. Recent advances in shear-band research focus on postmortem atomic structure characterization ${ }^{4}$ as well as direct probing of their dynamics during shear ${ }^{5,6}$ - a deformation regime that is key for understanding and controlling the often observed run-away failure associated with shear-band propagation.

When a shear band is initiated, both modelling and experiments suggest that a stress-induced glass transition occurs, where the material within the shear band is experiencing a volume dilatation equivalent to what one would expect for temperatures closely above the glass transition temperature. ${ }^{6,7}$ The formed layer has structurally softened after formation, now allowing thermally activated propagation to take place that mediates global plastic flow. ${ }^{5}$ The initiation process itself cannot be related to any corresponding global plastic strain, which is analogous to shear mechanisms observed in granular media. ${ }^{8}$

To date, the exact atomistic mechanisms of shear-band initiation in a metallic glass can only be studied using model systems, such as granular media ${ }^{9}$ or via modern computer simulations. ${ }^{10,11}$ In particular, molecular dynamics (MD) simulations have provided useful insights into how a shear band is potentially formed, and how the short-range and medium-range order of the matrix is changed during shearband initiation. Current findings revolve around the breaking-up and reduction of icosahedra motifs in the shear band that form a stiff back-bone in the matrix. ${ }^{11}$ Whilst this destruction of clusters and local order explains the structural softening, it remains unclear how the shear band is evolving into a planar defect spanning the material. Recent experimental work suggests a cross-over from three-dimensional

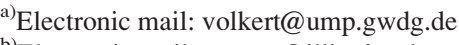

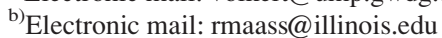

random activity to a two-dimensional nano shear band. ${ }^{12} \mathrm{~A}$ similar picture emerges from MD simulations, either indicating a propagating nucleation front mechanism, but also a merging of sites with locally reduced shear modulus and a cooperative activity of local plastic transitions at those sites may be envisioned as the initiation mechanisms. ${ }^{13}$ The later process is, in particular, interesting when considering the nanoscale spatial heterogeneity in amorphous metals that is believed to influence shear-band initiation. ${ }^{14}$ We note at this stage that MD straining experiments are studying athermal plasticity, which is at odds with the experimental evidence that deformation of MGs is thermally activated, which also applies for shear-band dynamics. ${ }^{5,15}$ Even though shearband initiation controls the onset of plasticity and therefore structural integrity, it remains a poorly understood problem in the field of metallic glasses.

In order to shed more light onto the formation stage of a shear band, we pursue instrumented nanoindentation measurements with different loading rates. In particular, we evaluate the local shear-stress distribution obtained from loads at which the first shear band is formed. More than 1000 measurements reveal that there is a clear rate dependence on the initiation stress of a shear band, which suggests thermally activated shear-band initiation. A thermal activation framework is used to extract the underlying range of activation volumes. In addition to the distribution of initiation stresses, a spatially correlated signature of elastic properties is revealed, showing systematic variations at the micron-scale. This structural signature is uncorrelated with the shear-band initiation stresses, leading to the conclusion that the rate dependent shear-band initiation process cannot be linked to the elastic heterogeneity of the volume probed under the indenter tip.

In this study, a $\mathrm{Pd}_{77.5} \mathrm{Cu}_{6} \mathrm{Si}_{16.5}$ bar was suction cast into a $\mathrm{Cu}$-mold. The bar was investigated with $\mathrm{x}$-ray diffraction $\left(\mathrm{Cu}-\mathrm{K}_{\text {alpha }}\right.$ line) to verify that a fully amorphous metal was produced. The as-cast surface of the sample with a root mean-square roughness of less than $1 \mathrm{~nm}$ was subjected to spherical nanoindentation at room temperature using an MTS (later Agilent/Keysight Technologies) Nano Indenter G200. 
The spherical diamond tip was calibrated on a fused silica reference standard, resulting in an effective tip radius of $\mathrm{R}=650 \mathrm{~nm}$. Nine arrays with $12 \times 12$ indents each (144 indents per array, separated by lateral distances of $8 \mu \mathrm{m}$ per indent to avoid overlap of the plastic regions) have been measured with different constant loading rates of $0.010 \mathrm{mN} / \mathrm{s}$, $0.025 \mathrm{mN} / \mathrm{s}, 0.100 \mathrm{mN} / \mathrm{s}$, and $0.250 \mathrm{mN} / \mathrm{s}$ to a maximum load of $0.4 \mathrm{mN}$ (that corresponds to a depth of $\sim 30 \mathrm{~nm}$ ). All load-displacement data were corrected for influence of thermal drift, machine compliance, and supporting spring stiffness. Figure 1 shows a typical load-displacement curve. The initial portion up to an indentation depth of $\sim 10 \mathrm{~nm}$ corresponds to purely elastic deformation, ${ }^{16}$ as given by the Hertzian elastic contact solution $^{17}$

$$
P=\frac{4}{3} E_{r} \sqrt{R} h^{1.5},
$$

with $P$ being the load, $E_{\mathrm{r}}$ is the reduced contact modulus, $R$ is the tip radius, and $h$ is the displacement. The blue solid line in Fig. 1 shows the exact solution. At indentation depths of around $10 \mathrm{~nm}$, a sudden displacement burst is visible, which is commonly referred to as a "pop-in". The very first pop-in marks the onset of plasticity that similarly to all consecutive pop-ins marks the formation of a shear band under the indenter tip. ${ }^{18}$ Using Hertzian contact theory, the stress condition that leads to the initiation of the first shear band can thus be studied by determination of the first deviation between the elastic solution and the recorded data.

In this study, the first pop-in of each test has been automatically detected with a custom written MATLAB script, which computes the indenter velocity point wise from the displacement and time data, and then detects pop-ins as spikes in the resulting velocity data. With that procedure, thousands of indents can be evaluated easily, and robust statistics about the onset of plastic deformation, that is shearband initiation, can be gained. We focus on the statistics of the maximum shear stress below the indenter at the onset of the first pop-in. This stress can be approximated by $\tau_{\max }$ $\approx 0.441 P_{\text {pop-in }} /\left(\pi R h_{\text {pop-in }}\right)$ with $P_{\text {pop-in }}$ and $h_{\text {pop-in }}$ being the load and displacement when the pop-in starts, and the pre-factor of 0.441 , which can be evaluated from the elastic contact framework ${ }^{16}$ with a Poisson's Ratio of $\nu=0.41$. In

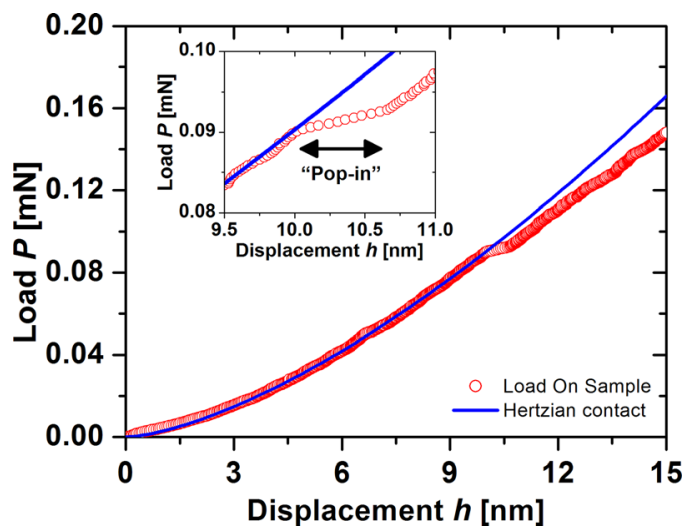

FIG. 1. A typical pop-in at the beginning of a load-displacement curve obtained with nanoindentation. The inset shows an enlarged view of the first pop-in typically occurring at $\sim 10 \mathrm{~nm}$ indentation depth.

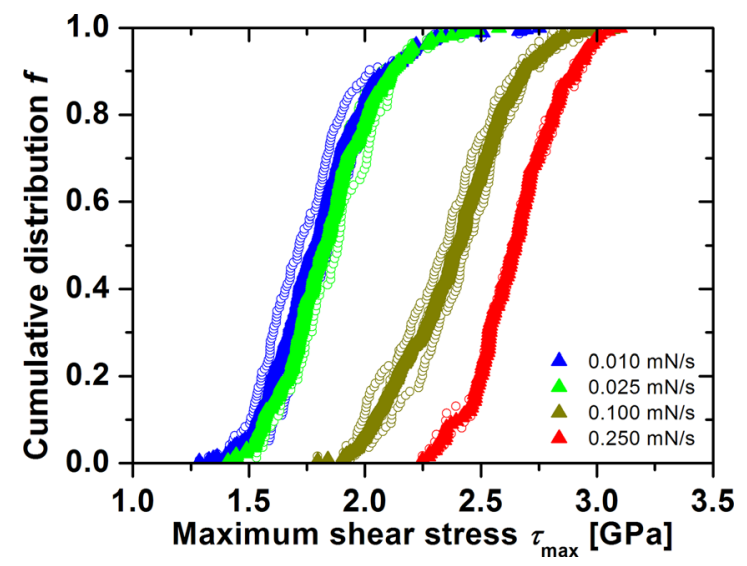

FIG. 2. Cumulative distributions of the maximum shear stress $\tau_{\max }$ at the first pop-in for different force rates. Open symbols stem from individual testing arrays, and full symbols are the merged distributions of several testing arrays.

Figure 2, the cumulative distributions, $f$, of $\tau_{\max }$ at the initiation of the first pop-in for each test of all the arrays tested at the four loading rates are shown. For each rate, open symbols summarize individual arrays, and full symbols are the cumulative distributions of several arrays tested at the same rate. It is seen that (i) $f$ remains robust when data of multiple arrays are merged, and that (ii) the general shape of the distributions (slope and tails) does not change much with rate. Furthermore, the position of the distributions scales strongly with the applied loading rate.

The spread of pop-in stresses over a considerable stress range in MGs has been observed and reported earlier by other researchers, ${ }^{19-22}$ but the effect of rate on $f$ remains a topic of controversy: Packard et al. ${ }^{19}$ and Wang et al. ${ }^{20}$ report rate-independent distributions for $\mathrm{Pd}-, \mathrm{Fe}-$, and $\mathrm{Zr}$ based MGs and conclude that the initiation of a shear band is primarily an athermally activated process. Instead, they propose that the spread in pop-in stresses rather stems from different structural configurations at the individual indentation sites. A similar suggestion was made by Perepezko et al., ${ }^{14}$ who reported a bi-modal cumulative distribution of a $\mathrm{Zr}$ based metallic glass, using only one fixed loading rate. On the other hand, the observation of a rate-dependent position of $f$ is scarce and was only made in Ref. 21 on a Zr-based MG. There are thus two different potential effects in Fig. 2: first, there is a spread in $\tau_{\max }$ due to structural variations across the amorphous material; and second, a rate-effect in the initiation stress of a shear band-the latter of which is a matter of dispute.

In order to better understand the structural influence on the shear-band initiation behavior, we need to investigate the possible structural heterogeneity in a robust way. This can be done by correlating the individual elastic properties of each indentation site to the maximum shear stresses at pop-ins. The Hertzian contact model is used to fit the data prior to the shear-band initiation stress. We performed an additional set of spherical nanoindentation tests at a loading rate of $0.025 \mathrm{mN} / \mathrm{s}$ on the same MG, which consists of four nested arrays of $10 \times 10$ indents each, with a lateral distance of $6 \mu \mathrm{m}$ between the indents. The arrays were placed on a lattice structure, such that array 2 was displaced by $(+3 \mu \mathrm{m}$, 
$0 \mu \mathrm{m})$ towards array 1 , array 3 was displaced by $(0 \mu \mathrm{m}$, $+3 \mu \mathrm{m})$ towards array 1 , and finally array 4 was displaced by $(+3 \mu \mathrm{m},+3 \mu \mathrm{m})$. Therefore, the total set of indents spans a resulting array of $20 \times 20$ indents and a covered area of $57 \times 57 \mu \mathrm{m}^{2}$ with an effective lateral distance between indents of $3 \mu \mathrm{m}$ in both directions (see left inset in Fig. 3 for a schematic). For these additional indentation sets, a maximum load of $0.2 \mathrm{mN}$ was used, which results in a typical indentation depth of $\sim 15 \mathrm{~nm}$ and a contact radius of $\sim 100 \mathrm{~nm}$. The reason for the nesting procedure was to ensure identification of any possible time-dependent and systematic errors in our measurements. The four arrays were measured at different times.

Figure 3 shows the evolution of the individual moduli for each of the four arrays as a function of distance along the indents. Since indents with identical test numbers from different arrays are spatially correlated (max. distance of $3 \mu \mathrm{m}$ ), one expects a similar trend as a function of test number for the different arrays if any long range correlations exist. Figure 3 shows that this clearly is the case: while the measured values do show some scatter, they follow the same trend, as seen by 7-point adjacent averaging (AAv, solid lines). The periodicity of ca. $120 \mu \mathrm{m}$ (20 indents) simply reflects the line pattern of the array (left inset Fig. 3), but what is remarkable is the fact that such long range correlations in local elastic properties can be identified. That means, the reported nano-scale fluctuations in elastic properties of $\mathrm{MGs}^{23}$ are here shown to also exist at a much larger length scale.

With the results of Fig. 3, we conclude that there is indeed a spatial heterogeneity of elastic properties in our tested MG that on the other hand does not correlate strongly with the corresponding maximum shear stress at the site of shear-band formation (right inset in Fig. 3). That means, the spread in $\tau_{\max }$ seen in Fig. 2 is not significantly influenced by the probed elastic properties at the testing site. This is not surprising keeping in mind that the elastic modulus is a value stemming from the elastic half sphere under the indenter, whereas the local shear transformations collectively mediating the formation of a shear band are more sensitive to structural heterogeneities at the atomic scale.

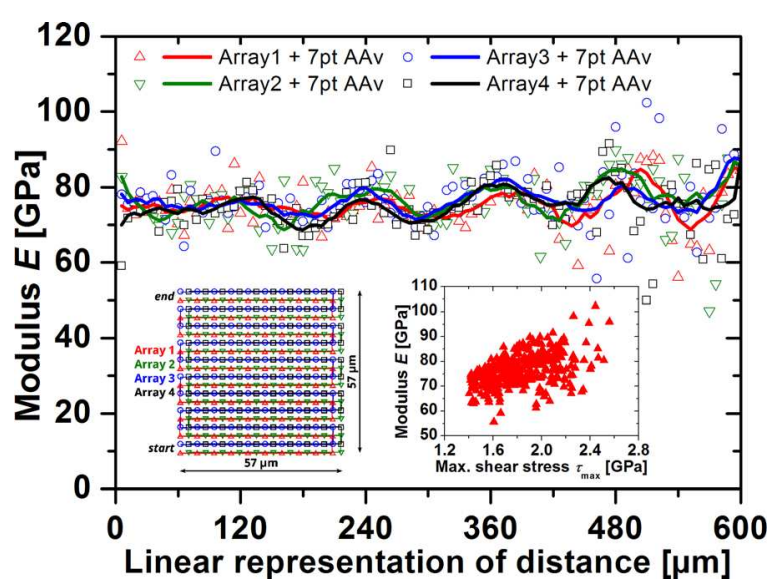

FIG. 3. Indentation modulus as a function of distance along the indent pattern for four different arrays. Open symbols are individual tests, full lines the 7pt AAv. The left inset schematically displays the nested array pattern. The right inset shows that $\boldsymbol{E}$ and $\tau_{\max }$ do not correlate strongly.

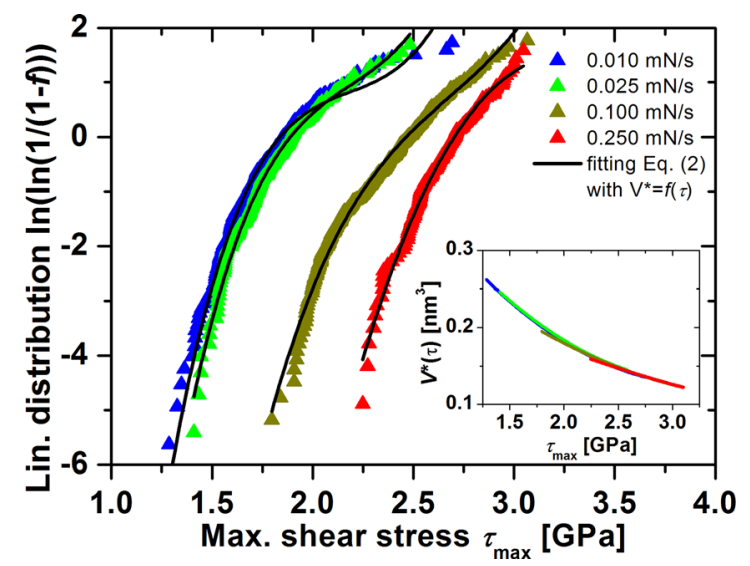

FIG. 4. The data in Figure 2 recast as $\ln (\ln (1 /(1-\boldsymbol{f})))$ as a function of $\tau_{\text {max }}$. Introducing $\boldsymbol{V}^{*}(\tau)=\boldsymbol{V}_{0}+\boldsymbol{V}_{1} \tau+\boldsymbol{V}_{2} \tau^{2}$ in Eq. (2) yields a good agreement with the data (solid black lines). The inset displays $\boldsymbol{V}^{*}(\tau)$ for the different indentation rates.

With the result at hand that MGs show local variations in elastic properties derived with nanoindentation, but that these cannot be directly correlated with the local stress distribution required for initiation of plasticity (Fig. 2), we turn our attention to the rate dependence revealed in Fig. 2. This trend with rate can be rationalized with a generic thermal activation model that has been proposed and applied to $\mathrm{SiC}$ single-crystals in Ref. 24 and subsequently was also used for nanoindentation data of a $\mathrm{Zr}$-based $\mathrm{MG}^{21}$ Fitting the data to the model would allow the determination of the activation volume for shear-band initiation. Before underlining the implications of the data seen in Fig. 2, we will outline the most relevant aspects of the used model. According to it, the cumulative distribution $f$ of thermally activated first popin shear-stresses $\tau$ (as in Fig. 2) can be derived via the differential equation $\dot{f}=(1-f) \dot{N}$ from the defect nucleation rate $\dot{N}=N_{0} \exp \left(-\frac{\Delta F^{*}-\tau V^{*}}{k T}\right)$. Here, $N_{0}$ is the atomic attempt frequency, $k T$ is the thermal energy, and the activation parameters are given by $\Delta F^{*}$, the activation free energy that is necessary to invoke a plastic process without stress assistance, and the activation volume $V^{*}$. After solving, $f$ follows the relationship:

$$
f=1-\exp \left(-\frac{N_{0} k T}{\dot{\tau} V^{*}} \times \exp \left(-\frac{\Delta F^{*}-\tau V^{*}}{k T}\right)\right),
$$

with $\dot{\tau}$ being the shear stress rate of the experiment. Equation (2) can be linearized as an $\ln (\ln (1 /(1-f)))$ vs. $\tau=\tau_{\max }$ plot, with the assumption that the inhomogeneous shear stress field under the indenter scales with the maximum shear stress $\tau_{\max }$. If we do so (Fig. 4), our distributions still line up in the same order as in Fig. 2. Opposite to the data set in Ref. 24 , our data clearly do not exhibit any linear behavior. Instead, one has to consider another approach to the data in Fig. 4 that appropriately captures the non-linear trend found in the experiments. Well motivated by thermally activated plasticity in MGs, ${ }^{25,26}$ we therefore interpret the data in Fig. 4 to be a result of a distribution of $V^{*}$.

In order to apply the model to our data and the underlying physical process, we hence suggest modifying Eq. (2). We extend Eq. (2) by the general notion in thermally activated 
plasticity that the activation volume depends on the stress, i.e., $V^{*}=f(\tau)$. Whilst the exact expression of $V^{*}(\tau)$ is not known, various different mathematical forms have been used. ${ }^{27,28} \mathrm{We}$ here choose to expand $V^{*}(\tau)$ by a simple quadratic power series $V^{*}(\tau)=V_{0}+V_{1} \tau+V_{2} \tau^{2}$ that falls back to the linear model if no stress dependence of $V^{*}$ is present. The parameters $V_{0}, V_{1}$, and $V_{2}$ give direct information about the cumulants of the assumed underlying distribution. By fixing the Helmholtz free energy at a typical value for shear transformation zone activation in MGs at $\Delta F^{*}=3 \mathrm{eV},{ }^{25}$ the temperature at $T=300 \mathrm{~K}$, and $N_{0}=10^{13} \mathrm{~s}^{-1}, \ln \left(\ln \left(1 /\left(1-f\left(V^{*}(\tau)\right)\right)\right)\right)$ was now evaluated at the different (maximum) shear stress rates $\dot{\tau}$. $\dot{\tau}$ was directly obtained from the four loading rates, amounting to $0.072 \mathrm{GPa} / \mathrm{s}, 0.18 \mathrm{GPa} / \mathrm{s}, 0.72 \mathrm{GPa} / \mathrm{s}$, and $1.8 \mathrm{GPa} / \mathrm{s}$. In all cases, the fits converge rapidly with an adjusted $\mathrm{R}^{2}$ greater than 0.98 , and a good description of the data shown in Fig. 4 is reached.

By using the fitting parameters $V_{0}, V_{1}$, and $V_{2}$, we display the resulting stress dependence of $V^{*}$, which is shown as an inset in Fig. 4. It shows the resulting quadratic power series within the region of their corresponding stress range of Fig. 4, demonstrating a very good agreement and consistency among the results for all four applied loading rates. The resulting values of the activation volume, ranging from 0.26 to $0.12 \mathrm{~nm}^{3}$, represent an apparent physical volume of ca. 18 to 8 atoms, which is very much in agreement with values found for other MGs, ${ }^{29}$ but we emphasize that instead of one mean value obtained from a poor linearization of the data in Fig. 2, we extract the range of activation volumes underlying the thermally activated shear-band initiation process. The trend of the decreasing activation volume with shear stress under the indenter observed in the inset of Fig. 4 is also in good agreement with the results of the aforementioned reports that assume a stress-dependent activation volume. $^{27,28}$ Before summarizing, it is noted that instead of assuming $V^{*}=f(\tau)$ and setting $\Delta F^{*}$ constant, one could also fix $V^{*}$ and assume $\Delta F^{*}=f(\tau)$. Fitting shows that this choice leads to a similar fitting quality but also the physically less intuitive result of a $\Delta F^{*}$ that monotonically increases with stress.

In summary, the statistical evaluation of pop-in behavior during nanoindentation experiments on an as-cast $\mathrm{Pd}_{77.5} \mathrm{Cu}_{6} \mathrm{Si}_{16.5}$ metallic glass strongly indicates that the plastic events underlying the pop-ins are activated by thermal fluctuations. Therefore, a wide spread in pop-in stresses of the order of $1 \mathrm{GPa}$ can be identified in the cumulative distributions. In order to properly quantify the activation parameters, a stress-dependent activation volume is used to fit the data. We conclude that the initiation of a shear band is increasingly confined to a more local activation volume (approximately a more local region) with rising stresses and rate. By evaluating an additional set of nanoindentation experiments, it is also revealed that the elastic properties vary spatially at the micrometer length scale. This elastic inhomogeneity does not correlate with the spread in pop-in stresses. The influence of elastic variations, as measured during nanoindentation of the glassy structure, seems therefore not to dictate the onset of shear-band formation because it probes a larger volume than what is structurally excited to initiate a shear band.

We are grateful to acknowledge experimental support by Uta Filippich (1st Physics Institute, University of Göttingen) during the sample production.

${ }^{1}$ L. Tian, Y.-Q. Cheng, Z.-W. Shan, J. Li, C.-C. Wang, X.-D. Han, J. Sun, and E. Ma, Nat. Commun. 3, 609 (2012).

${ }^{2}$ Y. Zhang and A. L. Greer, Appl. Phys. Lett. 89, 071907 (2006).

${ }^{3}$ R. Maass, K. Samwer, W. Arnold, and C. A. Volkert, Appl. Phys. Lett. 105, 171902 (2014).

${ }^{4}$ H. Rösner, M. Peterlechner, C. Kuebel, V. Schmidt, and G. Wilde, Ultramicroscopy 142, 1-9 (2014).

${ }^{5}$ R. Maass, D. Klaumünzer, and J. F. Löffler, Acta Mater. 59, 3205-3213 (2011).

${ }^{6}$ R. Maass and J. F. Löffler, Adv. Funct. Mater. 25, 2353 (2015).

${ }^{7}$ P. F. Guan, M. W. Chen, and T. Egami, Phys. Rev. Lett. 104, 205701 (2010).

${ }^{8}$ S. Nasuno, A. Kudrolli, A. Bak, and J. P. Gollub, Phys. Rev. E 58, 2161-2171 (1998).

${ }^{9}$ J. Török, S. Krishnamurthy, J. Kertesz, and S. Roux, Phys. Rev. Lett. 84, 3851-3854 (2000).

${ }^{10}$ E. R. Homer, Acta Mater. 63, 44-53 (2014).

${ }^{11}$ A. J. Cao, Y. Q. Cheng, and E. Ma, Acta Mater. 57, 5146-5155 (2009).

${ }^{12}$ J.-O. Krisponeit, S. Pitikaris, K. E. Avila, S. Kuechemann, A. Krueger, and K. Samwer, Nat. Commun. 5, 3616 (2014).

${ }^{13}$ A. L. Greer, Y. Q. Cheng, and E. Ma, Mater. Sci. Eng., R 74, 71-132 (2013).

${ }^{14}$ J. H. Perepezko, S. D. Imhoff, M.-W. Chen, J.-Q. Wang, and S. Gonzalez, Proc. Natl. Acad. Sci. U. S. A. 111, 3938-3942 (2014).

${ }^{15}$ R. Maass, D. Klaumünzer, G. Villard, P. M. Derlet, and J. F. Löffler, Appl. Phys. Lett. 100, 071904 (2012).

${ }^{16}$ H. Bei, Z. P. Lu, and E. P. George, Phys. Rev. Lett. 93, 125504 (2004).

${ }^{17}$ A. C. Fischer-Cripps, Nanoindentation, 1st ed. (Springer, New York, 2004).

${ }^{18}$ B. Moser, J. Kuebler, H. Meinhard, W. Muster, and J. Michler, Adv. Eng. Mater. 7, 388-392 (2005).

${ }^{19}$ C. E. Packard, O. Franke, E. R. Homer, and C. A. Schuh, J. Mater. Res. 25, 2251-2263 (2010).

${ }^{20}$ L. Wang, H. Bei, Y. F. Gao, Z. P. Lu, and T. G. Nieh, Acta Mater. 59, 7627-7633 (2011).

${ }^{21}$ I.-C. Choi, Y. Zhao, Y.-J. Kim, B.-G. Yoo, J.-Y. Suh, U. Ramamurty, and J.-i. Jang, Acta Mater. 60, 6862-6868 (2012).

${ }^{22}$ I.-C. Choi, Y. Zhao, B.-G. Yoo, Y.-J. Kim, J.-Y. Suh, U. Ramamurty, and J.-i. Jang, Scr. Mater. 66, 923-926 (2012).

${ }^{23}$ H. Wagner, D. Bedorf, S. Kuechemann, M. Schwabe, B. Zhang, W. Arnold, and K. Samwer, Nat. Mater. 10, 439-442 (2011).

${ }^{24}$ C. A. Schuh and A. C. Lund, J. Mater. Res. 19, 2152-2158 (2004).

${ }^{25}$ C. A. Schuh, T. C. Hufnagel, and U. Ramamurty, Acta Mater. 55, 4067-4109 (2007).

${ }^{26}$ P. M. Derlet and R. Maass, Philos. Mag. 93, 4232-4263 (2013).

${ }^{27}$ M. Schwabe, S. Kuchemann, H. Wagner, D. Bedorf, and K. Samwer, J. Non-Cryst. Solids 357, 490-493 (2011).

${ }^{28}$ D. Caillard and J. L. Martin, Pergamon Materials Series (Pergamon, 2003), Vol. 8, pp. 3-433.

${ }^{29}$ D. Pan, A. Inoue, T. Sakurai, and M. W. Chen, Proc. Natl. Acad. Sci. U. S. A. 105, 14769-14772 (2008). 